\title{
Es bleibt hypothetisch
}

\section{Dominique Eich-Höchli}

Prof. Dr. med., Leitende Ärztin Klinik für Psychiatrie, Psychotherapie und Psychosomatik, Spezialambulatorium ADHD, Psychiatrische Universitätsklinik Zürich

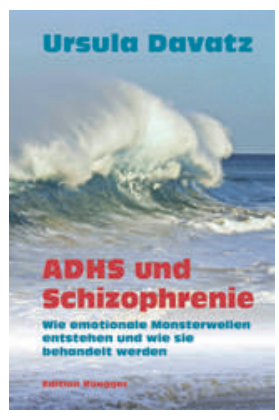

Ursula Davatz

ADHS und

Schizophrenie

Wie emotionale Monsterwellen entstehen

und wie sie behandelt werden

Glarus: Somedia

Buchverlag; 2014

320 Seiten. 44 CHF.

ISBN 978-3-7253-1020-3
Korrespondenz:

Prof. Dr.med.

Dominique Eich-Höchli

Psychiatrische

Universitätsklinik Zürich

Lenggstrasse 31

CH-8032 Zürich

dominique.eich[at]puk.zh.ch
Ursula Davatz schöpft in ihrem Buch ADHS und Schizophrenie aus dem Fundus von mehr als vierzig Jahren Berufserfahrung als Psychiaterin und systemische Familientherapeutin mit zahllosen Familien und den an Schizophrenie erkrankten Mitgliedern. An diesem Buch hat sie siebzehn Jahre gearbeitet, was Respekt verdient. Es ist die "Summa» ihrer engagierten therapeutischen Arbeit. Darin entwickelt sie anhand einer Vielzahl von familiären Stresssituationen unter systemischem Blickwinkel eine eigene Schizophrenie- Hypothese, die als ursächlichen Faktor das Entstehen einer «emotionalen Monsterwelle», analog einem «Tsunami», benennt.

Als Unterstützung für ihre Hypothese zitiert sie die Publikationen der Psychiatric Genomics Cross-Disorder Group 2012 (leider nicht in der Literaturliste aufgeführt), wo für fünf Krankheitsbilder (u.a. Schizophrenie und ADHS) angeblich dieselben genetischen Risikofaktoren bestehen. Allerdings wird in einer späteren $\mathrm{Pu}$ blikation der gleichen Gruppe 2013 [1] für diese fünf Störungen von "shared risk genes pathway" gesprochen (gemeinsamer Pfad für Risikogene), was etwas anderes bedeutet. Die Folgerung von Davatz, dass ADHS «die übergeordnete genetische Konstellation darstellt, die über eine spezifische Interaktion mit dem erzieherischen Umfeld zur Schizophrenie führen kann", bleibt hypothetisch. Zur Frage der Risikofaktoren für schizophrene Störungen gibt es viele Studien, die im Kindes- und Jugendalter unspezifische Symptome wie Ängste, Depressionen, aber auch die Störung des Sozialverhaltens (mit und ohne oppositionellem, aufsässigem Verhalten) nennen. Es ist diese Störung (und nicht ADHS), die in erster Linie der Entwicklung von psychiatrischen Störungen im Erwachsenenalter, unter anderem auch der Schizophrenie, vorausgeht. Hier muss betont werden, dass «vorausgehen» in keiner Weise mit «verursachen» verwechselt werden darf [2].

In den einzelnen Buchkapiteln finden sich viele Falldarstellungen aus der therapeutischen Tätigkeit der Autorin, die sie als Beleg für ihre Hypothese anführt. Allerdings wird die Aufmerksamkeitsdefizit-Hyperaktivitätsstörung (ADHS) im Kindesalter in der Flut der sehr knapp gehaltenen Fallvignetten kaum thematisiert. Es fehlen leider die Diagnosen (weder CD-10 noch DSM) und genauso retrospektive Angaben zur Anamnese der Betroffenen wie beispielsweise zur Entwicklungsgeschichte durch Angehörige oder andere Dritt- personen. Ihren Patientengeschichten gemeinsam ist die Tatsache, dass in Familiensystemen, wo eine Schizophrenie vorkommt, häufig nicht vollständig vollzogene Ablösungsprozesse beobachtet werden, die sich nach Davatz über mindestens drei Generationen erstrecken sollen. Die Autorin beschreibt eine festgefahrene Entwicklung, die aufgestaut als "emotionale Monsterwelle» über einem dafür vulnerablen Familienmitglied in einer akuten Psychose zusammenschlägt.

Unbestritten ist, dass in der Prodromalphase der Schizophrenie Störungen der Aufmerksamkeit (Ablenkung durch inneres Erleben u.a.m.) vorkommen. Diese können durchaus den Aufmerksamkeits- und Konzentrationsstörungen bei ADHS ähneln, sind aber syndromal völlig unterschiedlich einzuordnen. Aus der Parallelität des Vorkommens von gleichen Symptomen bei ADHS und Schizophrenie (deren ätiologische Ursache

\section{Aus der Parallelität des Vorkommens von} gleichen Symptomen bei ADHS und Schizophrenie darf nicht abgeleitet werden, dass ADHS eine Vorstufe der Schizophrenie ist.

bis heute ungeklärt ist) darf nicht abgeleitet werden, dass ADHS eine Vorstufe der Schizophrenie ist. Die wissenschaftliche Prüfung einer "shared vulnerability»Hypothese erfordert, neben einer langjährigen Verlaufsuntersuchung, eine aufwendige Methodik. Dies übersteigt bei weitem die kurzen Fallvignetten der Autorin, die zudem einen Untersucherbias beinhalten. Zusammenfassend: Der Titel ADHS und Schizophrenie weckt Erwartungen, die im Buch leider nicht eingelöst werden. Die Schwachstellen der Hypothesenbildung wurden oben bereits angeführt. Weiter ist bedauerlich, dass im Text zitierte Arbeiten sich im Literaturverzeichnis nicht wiederfinden.

Mit Gewinn liest dieses Buch, wer Interesse an biographisch unterlegter Psychiatriegeschichte, Spekulationen zur Schizophreniegenese und einer Vielzahl aus systemischer Sichtweise kommentierter Fallvignetten hat.

\section{Literatur}

1 Cross-Disorder Group of Psychiatric Genomics Consortium. Identification of risk loci with shared effects on five major psychiatric disorders: a genom-wide analysis. Lancet. 2013;381(9875):1371-9.

2 Kim-Cohen J, et. al. Prior Juvenile Diagnosis in Adults With Mental Disorder. Arch Gen Psychiatry. 2003;60:709-17. 